\section{Micro-RNA in der Pathogenese rheumatischer Erkrankungen}

\author{
S. Gay ${ }^{1}$ \\ ${ }^{1}$ Department of Rheumatology, University Hospital, Zurich, \\ Switzerland
}

Unser Forschungszentrum beschäftigte sich über die letzte Dekade intensiv mit den epigenetischen Regulationen der Expression von Genen. Zu diesen regulatorischen Prozessen gehört auch die Modulation durch microRNAs. Man darf in diesem Zusammenhang aber nicht übersehen, dass microRNAs sowohl durch Acetylierung als auch Methylierung reguliert werden [1].

Fakt ist, dass wir trotz der Anwendung neuer anti-rheumatischer Medikamente, hier müssen besonders die äußerst effektiven „Biologicals“ genannt werden, die Rheumatoide Arthritis (RA) oder progressive systemische Sklerose bisher nicht heilen können. Das liegt an der Tatsache, dass die synovialen Fibroblasten (SF) bei der RA bisher nur als Effektorzellen betrachtet wurden und nicht als aktivierte krankheitsunterhaltende Zellen therapeutisch angegangen wurden [2]. Das gleiche trifft für die Fibroblasten bei den fibrosierenden Erkrankungen zu.

In der Suche nach neuen therapeutischen Ansätzen haben wir uns deshalb in den letzten Jahren auch besonders den microRNAs ( $\mathrm{miR}$ ) zugewendet $[3,4]$.

So konnten wir zuerst zeigen, dass miR146a und miR155 in RASF und besonders miR155 auch in Monozyten stark exprimiert werden [5] und als proinflammatorischer Regulator in Arthritismodellen fungiert [6]. Wir konnten ferner nachweisen, dass miRs wie miR203 entscheidend die inflammatorischen Zytokine wie IL6 in RASF stimuliert und selbst durch Demethylierung induziert wird [7]. Wie komplex die Regulation dieser Prozesse ist, zeigt, dass miR18a nicht nur die IL6induzierte Produktion von den Akute-Phase-Proteinen Fibrinogen und Haptoglobin in humanen Hepatozyten reguliert [8], sondern selbst durch TNF $\alpha$ induziert wird und dabei RASF durch einen Feedback-loop im NFkB-Signaling aktiviert [9].

Den miRs kommt auch entwicklungsbiologisch eine wichtige Funktion zu. So konnten wir in kooperativen Untersuchungen mit der ETH in Zürich nachweisen, dass die Funktion der Desmosomen in Keratinozyten durch die Nrf2-miR29-desmocollin axis reguliert wird [10].

Dass antago-miRs auch therapeutisch eingesetzt werden können, konnten wir in einem Modell der Hypoxie-induzierten pulmonalen Hypertrophie beweisen [11].

Besonders attraktiv erscheint der geplante therapeutische Einsatz von antago-miR323, denn wir konnten miR323 als Regulator von TIMP3 nachweisen. TIMP3 kommt eine entscheidende Rolle in der Hemmung der Aktivierung der proinflammatorischen Zytokine IL6 und TNF $\alpha$ zu.
Anmerkung: Da diese Untersuchungen sehr viel teure Sachmittel und technologische Infrastrukturen erfordern, muss an dieser Stelle erwähnt werden, dass unsere Untersuchungen durch umfangreiche Förderung durch die EU finanziert worden sind (FP6 Autocure; FP7 Masterswitch; FP7 Osteoimmune; FP7 Euroteam; IMI BTCure) sowie das Institute of Arthritis Research in Epalinges $(\mathrm{CH})$.

Literatur

1 Kuchen S, Resch W, Yamane A et al. Regulation of microRNA expression and abundance during lymphopoiesis. Immunity 2010; 32: 828-839

2 Frank-Bertoncelj M, Gay S. The epigenome of synovial fibroblasts: an underestimated therapeutic target in rheumatoid arthritis. Arthritis Res Ther 2014; 16: 117

3 Filkova M, Jungel A, Gay RE et al. MicroRNA in rheumatoid arthritis: potential role in diagnosis and therapy BioDrugs 2012; 26: 131-141

4 Vettori S, Gay S, Distler O. Role of MicroRNAs in Fibrosis. Open Rheumatol J 2012; 6: 130-139

5 StanczykJ, Pedroli DM, Brentano Fet al. Altered expression of microRNA in the synovial fibroblasts and synovial tissue in rheumatoid arthritis. Arthritis Rheum 2008; 58: 1001-1009

6 Kurowska-Stolarska M, Alivernini S, Ballantine LE et al. MicroRNA-155 as a proinflammatory regulator in clinical and experimental arthritis. Proc Natl Acad Sci USA 2011; 108: 11193-11198

7 StanczykJ, Ospelt C, Karouzakis E et al. Altered expression of miR-203 in rheumatoid arthritis synovial fibroblasts and its role in fibroblast activation. Arthritis Rheum 2011; 63: 373-381

8 Brock M, Trenkmann M, Gay RE et al. MicroRNA-18a enhances the IL-6 mediated production of the acute-phase proteins fibrinogen and haptoglobin in human hepatocytes. J Biol Chem 2011; 286: 40142-40150

9 Trenkmann M, Brock M, Gay RE et al. The TNFo-induced miR-18a activates rheumatoid arthritis synovial fibroblasts through a feedback loop in NF-KB signaling. Arthritis Rheum 2013; 65: 916-927

10 Kurinna S, Schäfer M, Ostano Pet al. A novel Nrf2-miR-29-desmocollin-2 axis regulates desmosome function in keratinocytes. Nat Commun 2014; 5: 5099

11 Brock M, Samillan VJ, Trenkmann $M$ et al. AntagomiR directed against miR-20a restores functional BMPR2 signalling and prevents vascular remodelling in hypoxia-induced pulmonary hypertension. Eur Heart J 2014; 35: 3203-3211

\section{Bibliografie}

DOI http://dx.doi.org/10.1055/s-0035-1558066

Drug Res 2015; 65, Suppl. 1: S18-S18

(c) Georg Thieme Verlag KG Stuttgart · New York .

ISSN 2194-9379

Korrespondenzadresse

\section{Prof. Dr. Steffen Gay}

Center of Experimental Rheumatology

Universitätsspital Zürich

Gloriastr. 25

8091 Zürich

Schweiz

steffen.gay@usz.ch

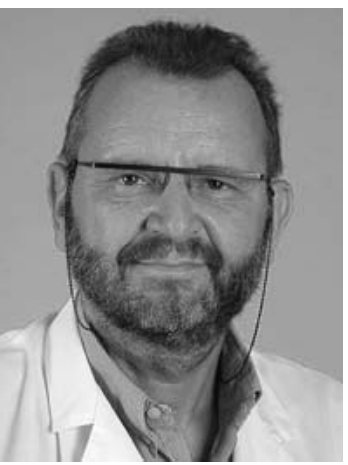

